\title{
Multi-sensor system using plastic optical fiber for intrinsically safe level measurements
}

\author{
C. Vázquez*, A.B. Gonzalo, S. Vargas, J. Montalvo \\ Dpto. Tecnología Electrónica, Escuela Politécnica Superior, Universidad Carlos III Madrid Avenida Universidad 30, \\ Leganés, Madrid 28911, Spain
}

\begin{abstract}
A system for measuring liquid level in multiple tanks using optical fibe technology has been developed. Oil fiel service industry or any sector requiring liquid level measurements in flammabl atmospheres can be benefite from this intrinsically safe technology. The device used a single lens for the emitting and receiving fibe and it is based on amplitude variations as a function of the liquid distance and not in time of fligh or phase detection. Being the firs fiber-opti liquid level sensor with those characteristics for long ranges ( $>200 \mathrm{~cm})$. A simple model to describe their behavior has been derived and tested on two prototypes. A Monte-Carlo method is used to fi the experimental data and obtain the model parameters. High accuracy between experimental data and fitte curve is obtained. The prototypes have a good linearity, better than $1.5 \%$ FS (full scale). Sensor heads are made of plastic optical fiber (POF) that are easy to handle, fl xible and economical. They are excited by $650 \mathrm{~nm}$ lasers, housed in ST-connectors to obtain compact and rough prototypes. Optical multiplexing is used to increase the measuring safety area. Frequency division multiplexing is used to address each sensor head. A discussion about the influenc of tilts and aberrations is also included.
\end{abstract}

Keywords: Fiber optics; Liquid level measurement; Sensors; Oil industry

\section{Introduction}

Intrinsically safe measurements are critical in a wide range of industrial environments such as flammabl atmospheres. Nowadays, a visual inspection of fuel level in open tanks is carried out in many petrol stations. But, volatile organic compounds (VOC) are emitted while opening the tanks, deteriorating ozone layer and being able to hurt operator health. Optical technologies with optical fiber are intrinsically safe in nature, with no risk of explosion even under malfunction operation, because inside the tank and the surroundings there are only inert materials such as optical fiber [1]. Different laser and optical instrumentation devices have been already used in level measurement systems, as the level gauge described in [2] and the references included in this patent; but in any of them the laser, so the electronic driver, is in the sensor head. Optical fiber have been used in the sensor heads for measuring very short distances, in the micrometer range with a high precision, using fiber-opti long period gratings as in [3], for measuring tens of centimeters employing: continuous flui sensing fiber [4-6], or intrusive large arrays of individual fluid-sensit ve transducers [7]. Another works on liquid level sensors reported intrusive digital transducers with a range of several meters using fiber with clad and unclad zones [8] or liquid-coupling waveguides [9], microbending optical-fibe sensors with hysteresis for indirectly measuring up to $2.5 \mathrm{~m}$ [10] and transducers for point measurements in control level devices [11-13]. A remote sensor head based on optical fiber for long distances, non-intrusive and without hysteresis is presented in [14] but using different lenses for transmitter and receiver fiber and the level in the vessel is measured as a function of the time required for a laser signal to be transmitted from the sensor, reflecte off the liquid surface and returned to the receiver lens located in the sensor.

This paper reports a multi-sensor system for level measurements, which is based, on new plastic optical fibe (POF) continuous, non-intrusive, liquid level sensors with no hysteresis. A theoretical model describing its behavior is also shown. It is a simple design using an unique lens to collimate and focus the light and it is based on amplitude variations as a function of the liquid distance and not in time of fligh or phase detection. Being the firs fiber-opti 
liquid level sensor with those characteristics. The use of POFs, which are easy to handle, fl xible and inexpensive allows developing inexpensive sensor heads, as in other POF sensors [15]. Multiple tanks are addressed using frequency division multiplexing (FDM) and optical fibe combiners. Optoelectronics circuits are developed for properly conditioning the laser output and the light reflecte off the liquid surface. Control and data acquisition in the system is developed using a microcontroller, which is connected, via RS-232 port to the PC. Measurements on two prototypes are developed for validating purposes.

The paper is focused on the development of the novel sensors and their multiplexing and it is structured as follows. Section 2 presents the schematic of the sensor heads, principle of operation and models describing its behavior. In Section 3, a description of the multi-sensor system is given along with the electronics for a proper operation. Section 4 is devoted to test on the prototype and validation of the model. A discussion is reported in Section 5. Finally, Section 6 elaborates on the summary and conclusions.

\section{Principle of operation and sensor heads modeling}

The attenuation of the light transmitted from the sensor, reflecte off the liquid surface and returned to the receiver fibe depends on the distance from the sensor head to the liquid surface. A unique lens is used to collimate the incident beam and to focus the reflecte beam.

\subsection{Simple model}

The emitter fibe is placed in the lens' focal plane, near the focus and it is considered as a point source. Therefore, behind the lens, there is a collimated beam that is tilted a little angle, referred to the lens optical axis. The beam is reflecte by the liquid surface and comes back to the lens (see discontinuous trace in Fig. 1). Due to the mentioned angle, the beam suffers a lateral displacement that depends on the distance between the lens and the liquid surface, $D$. Consequently; the lens collects only a part of the beam, whose

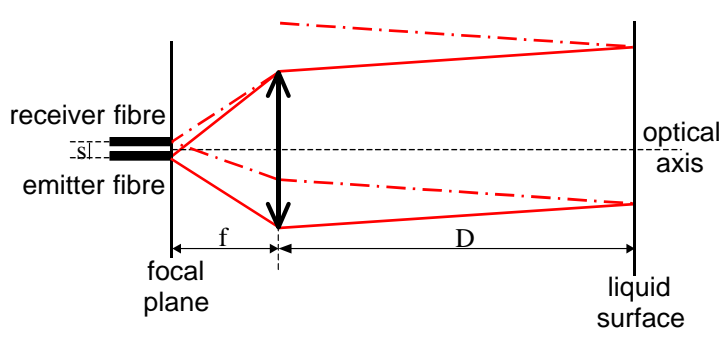

Fig. 1. A schematic showing the incident (-) and reflecte (---) beam path depending on the location of the lens and the emitter and receiver fibers Some parameters that characterize the sensor are shown: $f=$ the lens focus, $D=$ distance between the lens and the liquid surface, $s=$ the distance between the fibe centers.

image is formed on the focal plane, in a point symmetric to the emitter fibe in relation to the focus. The receptor fibe is placed in this position. Assuming that:

- the absorption of the laser radiation by the air is negligible in the considered distance range,

- the emitter fibe is placed in a way that its numerical aperture does not produce any limitation,

- the power is uniformly distributed in the laser cross section;

the signal generated by the photodiode, which is placed behind the receptor fibe should be proportional to the rate of the common area, $S_{\text {com }}$, between the reflecte beam and the lens, to the total area of the reflecte beam, $S_{\text {tot }}$. As the emitter fibe is very close to the lens focus, the cross section of the reflecte beam could be treated as a circumference. If the beam is well collimated, the radius of the lens, $a_{1}$, and the reflecte beam, $a_{2}$, should be the same. In order to permit a small beam divergence, the radii are related as:

$a_{2}=a_{1}+2 D \operatorname{tg} \alpha$

where $\alpha$ is the beam divergence. Then, there are four possible situations, shown in Fig. 2:

(a) The two circumferences intersect. In this case, the distance between the centers of the circumferences, $c$, is limited by: $\left|a_{1}-a_{2}\right|<c<a_{1}+a_{2}$. Being $f$ the lens focus, $s$ the distance between the fibe centers and $D$ the distance between the lens and the liquid surface, $S_{\text {com }}$ is given by

$S_{\mathrm{com}}=\frac{1}{2} a_{1}^{2}\left(\theta_{1}-\sin \theta_{1}\right)+\frac{1}{2} a_{2}^{2}\left(\theta_{2}-\sin \theta_{2}\right)$

where

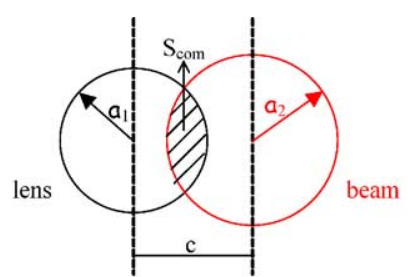

(a)

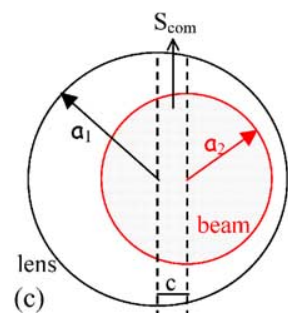

Fig. 2. Common area between the lens and the reflecte beam in the four possible situations. Some parameters that characterize the sensor are shown: $a_{1}=$ the lens radius, $a_{2}=$ the beam radius, $c=$ distance between circumferences centers. (a) Intersecting circumferences; (b) reflecte beam with positive divergence, $\alpha>0$; (c) reflecte beam with negative divergence, $\alpha<0$; (d) circumferences without common surface. 


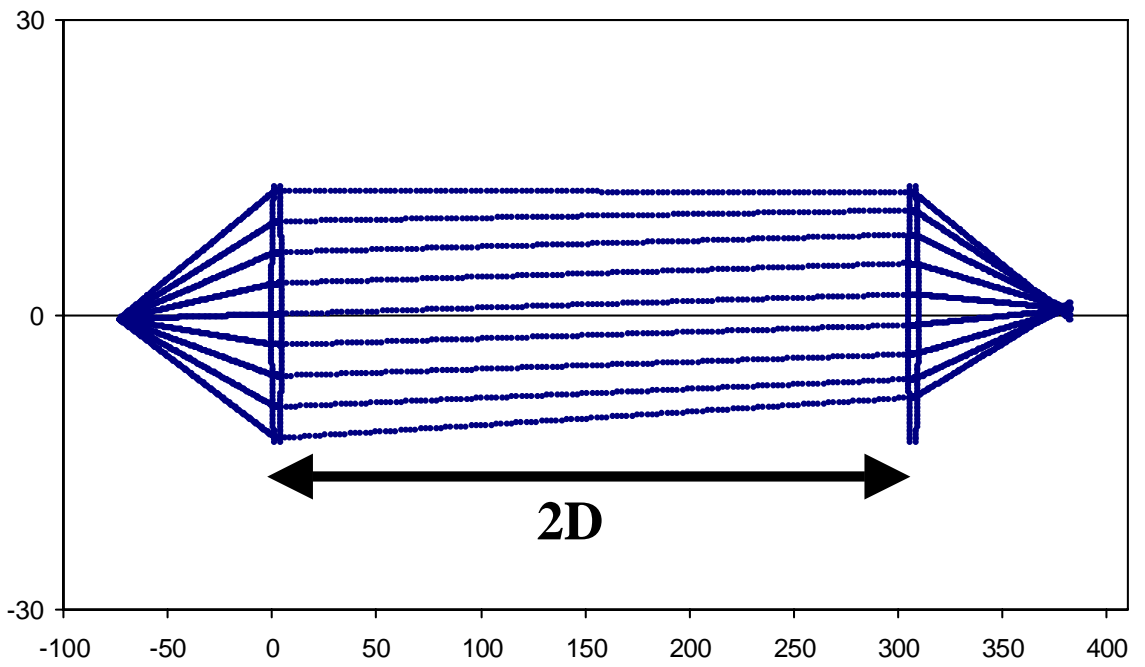

Fig. 3. Real propagation of the beam considering a lens focal $=75 \mathrm{~mm}$, and a lens diameter $=25.4 \mathrm{~mm}$. The graph's axis are in $\mathrm{mm}$.

$\theta_{1}=\pi-2 \arcsin \left(\frac{x_{\mathrm{C}}}{a_{1}}\right)$,

$\theta_{2}=\pi-2 \arcsin \left(\frac{c-x_{\mathrm{C}}}{a_{2}}\right)$,

$x_{\mathrm{C}}=\frac{a_{1}^{2}-a_{2}^{2}+c^{2}}{2 c}, \quad c=s \frac{D}{f}$

Therefore, we defin the function $f(k, \alpha)$ for describing the behavior of the sensor. This function is given by

$f(k, \alpha)=k \frac{a_{1}^{2}\left(\theta_{1}-\sin \theta_{1}\right)+a_{2}^{2}\left(\theta_{2}-\sin \theta_{2}\right)}{2 \pi a_{2}^{2}}$

where $k$ is a constant that includes the effects of the liquid reflectance fibe attenuation, etc. And $a_{1}, a_{2}, \theta_{1}$, $\theta_{2}$ are given by Eqs. (1) and (3).

(b) The lens radius is smaller than the beam one (slightly divergent beam) and the distance between the circumferences centers, $c$, has a value that holds: $c<a_{2}-a_{1}$. In this case, the function $f(k, \alpha)$ is calculated as follows:

$f(k, \alpha)=k \frac{a_{1}^{2}}{a_{2}^{2}}$

(c) The beam radius has a smaller value than the lens radius (slightly convergent beam) and the beam circumference is contained inside the lens one, that is, $c<a_{1}-a_{2}$. Function $f(k, \alpha)$ is given by

$f(k, \alpha)=k$

(d) The two circumferences do not intersect neither are one inside the other $\left(c>a_{1}+a_{2}\right)$. In this case there is no common surface, and $f(k, \alpha)$ is

$f(k, \alpha)=0$

We have fitte our experimental data to this function using the Monte-Carlo method. The algorithm looks for the parameters $k$ and $\alpha$ that minimize the "mean distance" between the experimental data and $f(k, \alpha)$. This "mean distance" is define as follows:

$d_{\mathrm{m}}=\sqrt{\frac{\sum_{i}(\operatorname{data}(i)-f(k, \alpha)(i))^{2}}{N}}$

with $N=$ number of data.

\subsection{Lens aberrations}

In order to evaluate the influenc of aberrations, the real propagation of the beam, from one fibe to the other, was simulated. To simplify the calculations, the liquid surface was eliminated, and the lens was duplicated. The emitter fibe was placed before the firs lens, an the receiver one after the second lens, as it can be seen in Fig. 3. Obviously, if $D$ is the lens-liquid distance, the distance between the two lenses is $2 D$. A fi ed number of rays $\left(R_{1} \approx 400000\right)$ leave the emitter fibe and impact on the firs lens, considering an uniform energy distribution on the emitter fibe. The direction of propagation of each ray trough the optical system is calculated using the Snell law. Then it is counted the rays that, crossing the second lens impact on the receiver fibe $\left(R_{2}\right)$. The quotient between $R_{2}$ and $R_{1}$ should be proportional to the sensor signal. It can be seen in Figs. 3

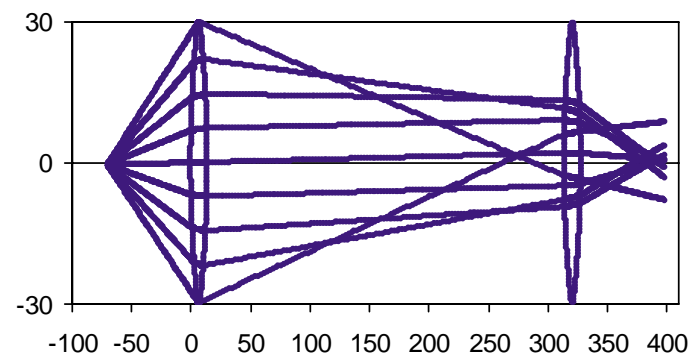

Fig. 4. Real propagation of the beam considering a lens focal $=75 \mathrm{~mm}$, and lens diameter $=60 \mathrm{~mm}$. The graph's axis are in $\mathrm{mm}$. 

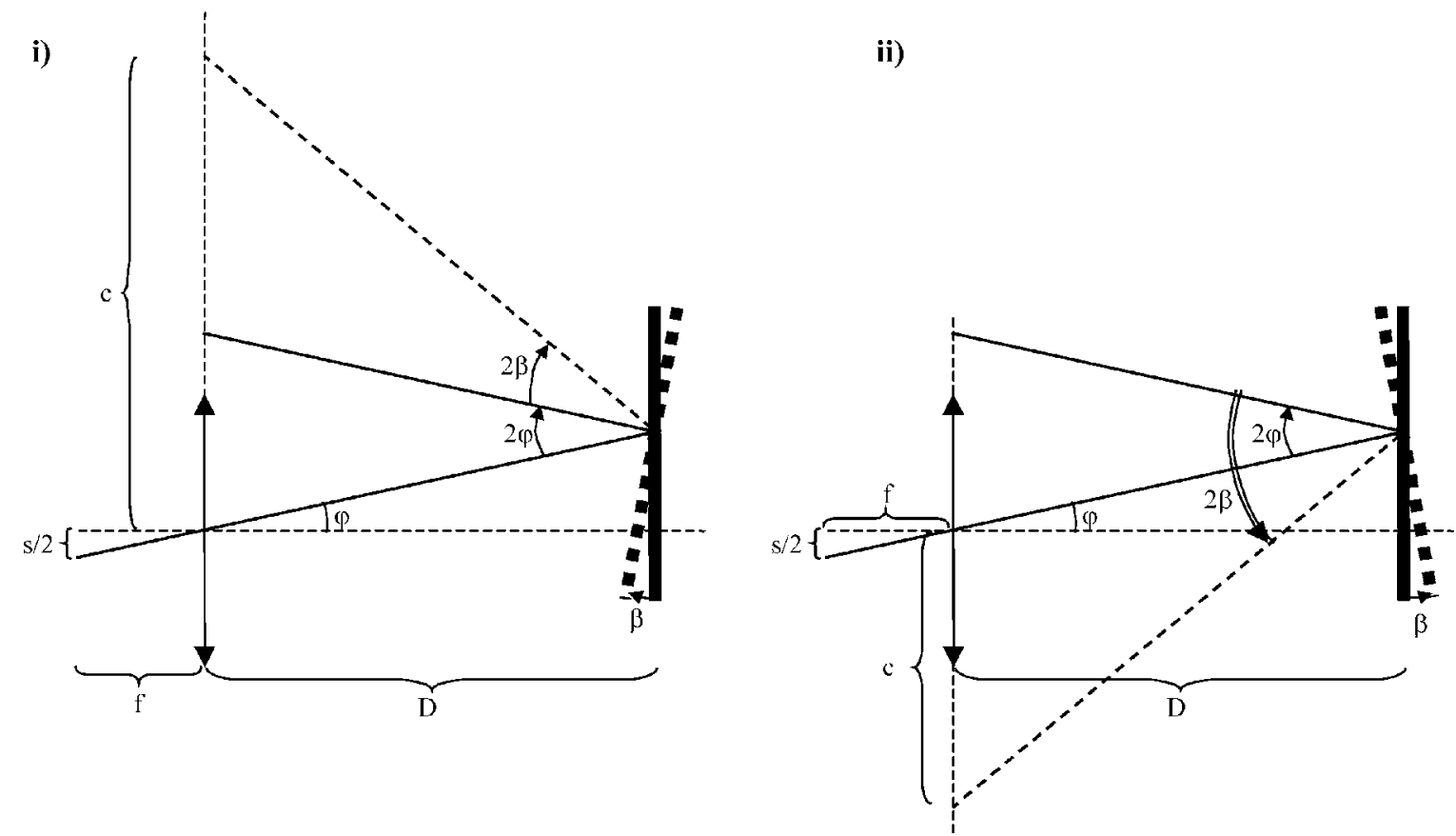

Fig. 5. Additional lateral displacement of the reflecte beam caused by a surface tilt: (i) surface tilt clockwise; (ii) surface tilt counterclockwise.

and 4, a couple of examples of the real propagation of the beam. In the firs one, the lens has a diameter of $25.4 \mathrm{~mm}$, and in the second one, the diameter of the lens is $60 \mathrm{~mm}$. The focal length is $75 \mathrm{~mm}$ in both cases. As it can be noted, in the second case, the beam energy distribution is affected by the aberrations stronger than in the firs case, because the system has higher apertures.

\subsection{Tilt modeling}

The liquid surface state has a great influenc on the sensor response. In the proposed model, a tilt of the liquid surface leads to an additional lateral displacement of the reflecte beam. This displacement can be calculated taking into account the known fact that, when a plane mirror is tilted an angle $\beta$, the reflecte ray is tilted an angle $2 \beta$ in the same direction. Depending on the tilt direction two situations can be considered. These are shown in Fig. 5, where it has been only represented the ray that crosses the lens center without suffering deviation, because this ray determines the position of the center of the back reflecte beam circumference.

Considering that the angles $\beta$ (surface tilt) and $\varphi$ (ray tilt) have small values, the new value for $c$, that is, for the distance between the centers of the two circumferences, in the case (i) is given by

$c=s \frac{D}{f}+\operatorname{Dtg}(2 \beta)$

And in the case (ii), the $c$ value can be calculated as

$c=\operatorname{Dtg}\left(\left|2 \beta-\frac{s}{f}\right|\right)$
Hence, we should use now this new expressions for $c$ in the proposed model for taking into account the surface tilt. However, if the sensor behavior is given by the case (b) of the model, that is, the radius of the reflecte beam is higher than the lens radius, and the lens surface is contained into the beam surface, the beam could be displaced laterally a certain quantity without modifying the sensor response. In this particular situation, it has been estimated that for liquid surface tilts less than the half value of the beam divergence, there is no influenc on the sensor response. However, for higher values the tilt of the liquid surface has fatal consequences not only for the model, but also for the sensor itself, which begin to not work properly. These considerations are well suited for the built sensor, because it works according to case (b) of the model.

On the other hand, different behaviors can be expected depending on the lens parameters. So two sensor heads with different lenses have been developed and the results are discussed in Section 4.

\section{Multi-sensor system architecture and implementation}

A schematic of the multi-sensor, scalable system can be seen in Fig. 6. Optical fiber are used in the sensor heads and for optical multiplexing to address different tanks without using multiple cables. This system allows an intrinsically safe level measurement with a simple, modular and cost effective solution. In the following we are going to describe in a firs approach the general architecture, and afterwards a brief description of the different 


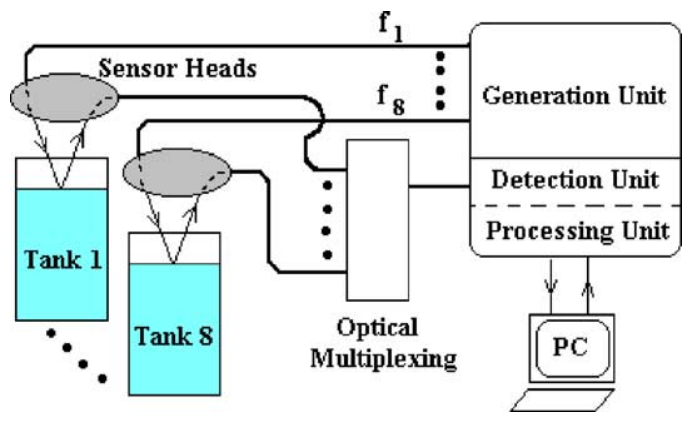

Fig. 6. A schematic of the multi-sensor system and its connection to the tanks.

blocks. A detailed description of the electronics is reported in [16]. Design and development aspects will be covered.

\subsection{System architecture}

The light signal used to measure the level is emitted by the generation unit, transmitted to the tank and collected after being reflecte off the liquid surface by the sensor heads. It is converted to the electrical domain by the processing unit to properly extract the desired information and adequate the signals to the microcontroller. These signals are sent to the Personal Computer $P C$, via RS232 port. A software application is developed to configur the system and show the measurement results. Scalability of the system is reached using frequency division multiplexing technique for addressing the signals of the multiple sensors. Each sensor head uses a laser diode modulated at a specifi frequency, after being reflecte off the liquid surface they are optically multiplexed in a single fibe, using $1 \times N$ POF couplers, and transmitted to the processing unit.

\subsection{Blocks description}

\subsubsection{Sensor heads}

They are made of the emitter and receiver fibers Both are PMMA POF with a $1 \mathrm{~mm}$ diameter and a 0.47 numerical aperture (NA). Having an attenuation of $0.24 \mathrm{~dB} / \mathrm{m}$ at $650 \mathrm{~nm}$. This choice was due to economical and practical reasons. POFs are cheaper and easier to handle than glass fibers their large core diameters allow greater manufacture tolerances and their high NA allow that a greater amount of light can be collected, increasing the sensor sensitivity.

They also include the collimating/focusing lens and the mechanical parts to align the sensors perpendicular to the liquid surface. In our design we have used a fibe receptacle base, which is supported by a base surface in "L" shape where the lens is placed in front of the fiber [17]. The lens determines the range and sensitivity of each sensor. The photograph of the two sensors heads are shown in Fig. 7. These sensors have lenses with different focal length and diameter and are made of different materials.

\subsubsection{Generation, detection and processing unit (GDP unit)}

Generation circuits include the laser diodes, S6505MG ( $5 \mathrm{~mW}$ maximum output power), that we have housed in metal ST-connectors (see Fig. 8) using the thermal bonding compound TBS from Electrolube. This compound has an excellent tensile strength, a very good thermal conductivity and excellent electric insulation characteristics. The generation circuits have also the laser diode drivers, which modulate the output signal and stabilize the average power.

The detection includes the reception and the demodulation circuits and it is designed for having a single detection stage for the multi-sensor system. The reception circuit has the optical to electrical (OE) converter, using a photodiode IF D91 housed in a "connector-less" style POF package with a $0.2 \mu \mathrm{A} / \mu \mathrm{W}$ responsivity at $632 \mathrm{~nm}$ and a rise and fall times

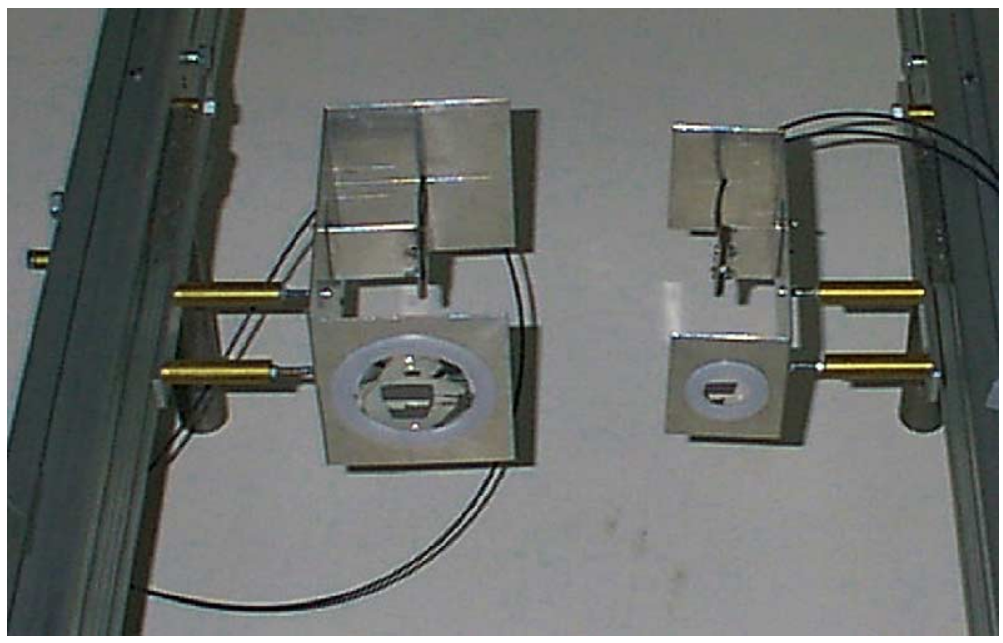

Fig. 7. Photograph of two sensor heads. 


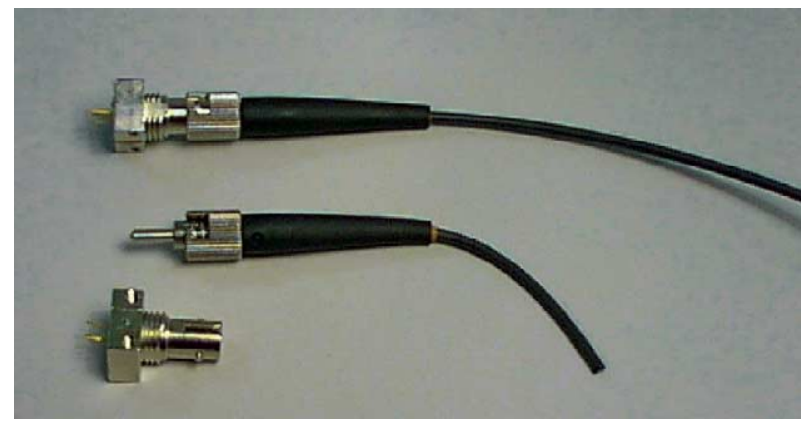

Fig. 8. Photograph of an ST fibe pigtail and the laser housed in an ST-connector is shown.

of $5 \mathrm{~ns}$. The demodulation circuit is the responsible to extract the information of each sensor. There is also a logarithmic stage for having a linear calibration curve, because there is an almost exponential dependence from the measured output voltage and the liquid level in the tank. Finally, an offset and a variable gain stage are implemented for adjusting the output voltage range in the liquid level measuring range.

The processing unit includes the acquisition system that converts the analog signals from the demodulator to the digital input signals to the microcontroller. Their basic functions are controlling the system and the communication with the PC. Controlling actions includes:

- laser diode drivers ON and OFF control;

- to decide the sampling sequence of the different sensors and to control its execution;

- controlling the number of measurements to be taken in each sensor.

\subsection{3. $P C$ and software application}

Configuratio of the system, data reception, post-processing and monitoring are done in the PC. An user-friendly software interface is developed in Visual Basic to show, in real time, the level in the different tanks and the alarms activation in case of surpassing predetermined risk levels.

\subsubsection{Optical multiplexing and transmission}

Scalability of the system is achieved using FDM technique. Allowing recombination of all the light signals in the optical domain for transmitting them to the GDP unit using a single optical fibe, so the intrinsically safe area is increased. Different alternatives have been considered; one uses $2 \times 1$ devices in cascade to multiplex all the sensor heads. The other proposed option uses $2 \times 1$ and $4 \times 1$ devices. The output single fibe is long enough to connect the whole sensor heads with the GDP unit. In typical petrol stations, the link will be of some meters.

\section{Experimental results}

In the following, we are going to describe the experimental set-up, the measured calibration curve and the static characteristics of the transducer. The different tests that have been carried out in the prototypes are also included. The experimental measurements have been used for validating the model and the results are also reported here.

\subsection{Experimental set-up}

A test-bench has been developed for allocating the sensor heads and aligning them perpendicularly to the liquid surface. It is made of aluminum rods fi ed on the laboratory walls from the floo to the ceiling. They can be partially seen in Fig. 7. A rule is stuck on the lateral of the aluminum rod for calibration purposes. Test-bench limitations have conditioned the calibration of the prototype at a $2 \mathrm{~m}$ range. Emitter and receiver fiber are connected to the electronic circuits and measurements are taken on the oscilloscope TDS210 and the PC. The measurement set-up is automatically controlled through HPVEE.

\subsection{Calibration curve and static characteristics}

Laser diode drivers operation have been tested. To do so, apart from measuring its bandwidth, it has been measured the average and peak-to-peak output power during $12 \mathrm{~h}$. The results show a $1.3 \%$ deviation in the average power and a maximum 3\% deviation in the peak-to-peak power during the $12 \mathrm{~h}$ test period. It has been measured that losses in the laser diode output for being embedded in metal ST-connectors are of $1.7 \mathrm{~dB}$.

Two different sensor heads (see Fig. 7) have been calibrated using our multi-sensor system. To do so, we have adjusted both sensors. The minimum output of the system in the small sensor at $207 \mathrm{~cm}$ is set to $0.5 \mathrm{~V}$ with the offset circuit. The maximum output of the small sensor is set to $5 \mathrm{~V}$, modifying the gain stage, for a $17 \mathrm{~cm}$ distance. Measurements have been averaged in the oscilloscope TDS210, having a greater number of samples in the longer distances. The measured calibration curves can be seen in Fig. 9. We see that the logarithmic stage effectively produces a linear

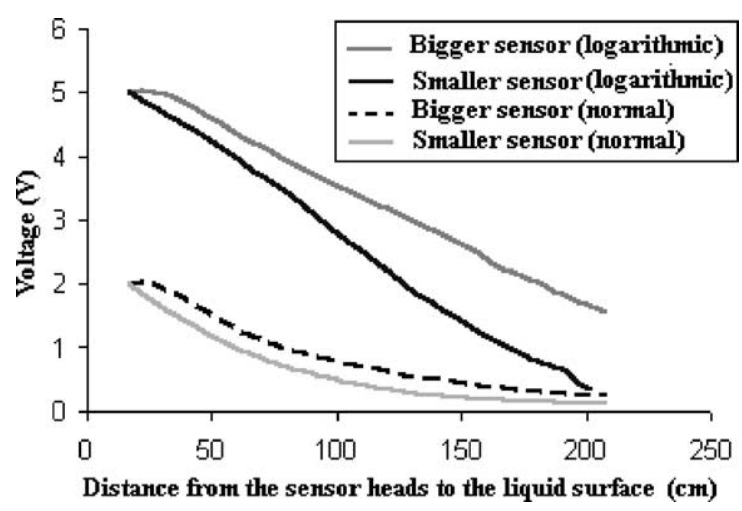

Fig. 9. Calibration curves before and after the logarithmic stage for two sensor heads after the demodulation stage. 
output voltage. On the other hand, as it was expected, the big sensor (having a bigger lens diameter) has a smaller slope, because can collect much more light at the same distance (see Fig. 2) and the maximum output voltage (5 V) was adjusted for the other sensor head. Linearity is worsened for the firs measurements up to around $20 \mathrm{~cm}$. On a second calibration in the big sensor head we have adjusted the output range for that sensor from 0 to $5 \mathrm{~V}$. In the new calibration curve a straight line has been fitte to the measured output voltage over the span of $37-207 \mathrm{~cm}$. System linearity is calculated as the maximum deviation between actual and linear predicted output voltage. Linearity is better than $1.5 \%$ FS from 37 to $207 \mathrm{~cm}$. For that span a system sensitivity of $0.02 \mathrm{~V} / \mathrm{cm}$ is obtained. During calibration the liquid surface must be quiet, small fluctuation can be compensated through averaging but brusque stirring will provoke wrong measurements.

As previously mentioned, test-bench limitations have conditioned the $2 \mathrm{~m}$ range. The output power at $207 \mathrm{~cm}$ is high enough to allow increasing the measuring range.

System Resolution of the full system is limited by AD conversion so it can be improved by employing a better conditioning. In the actual design with an 8 bit AD converter for the $2 \mathrm{~m}$ range a resolution $<0.5 \% \mathrm{FS}$ is obtained.

The system is developed for multiplexing eight sensors, but only optical multiplexing of three sensors heads have been carried out. A $3 \times 1$ POF coupler is used in the optical multiplexing and spectral measurements of the output voltage are reported in Fig. 10. Metal connectors are used
Table 1

Parameters of the simple model obtained in the fi using the Monte-Carlo method for the two sensor heads

\begin{tabular}{llll}
\hline Lens diameter $(\mathrm{mm})$ & $d_{\mathrm{m}}$ & $\alpha(\mathrm{rad})$ & $k$ \\
\hline 60 & 0.031 & 0.020 & 4.040 \\
25.4 & 0.052 & 0.014 & 4.988 \\
\hline
\end{tabular}

to connect the receiver fibe pigtails and the POF coupler input. This figur shows a photograph of the TD210 oscilloscope screen operating in its FFT mode. It can be seen that there is no interference between the signals of the different sensors, which are modulated at frequencies around $10 \mathrm{kHz}$ to avoid DC noise.

\subsection{Model validation}

Eq. (8) has been used to fi the simple model parameters for both sensors from measured calibration curves prior to the logarithmic stage. Results of the fittin process and experimental data can be seen in Fig. 11. It is plotted the sensor output signal in volts versus the liquid-lens distance in $\mathrm{mm}$. The circles correspond to the measurements performed with a $60 \mathrm{~mm}$ diameter lens and the crosses to a $25.4 \mathrm{~mm}$ diameter lens. The parameters obtained in the fit are shown in Table 1. They have a low quadratic dispersion. It can be seen that the "mean distance" between measured curves and theoretical model, $d_{\mathrm{m}}$, is very small. In the case of the bigger lens, $d_{\mathrm{m}}$ is the $3 \%$ of the

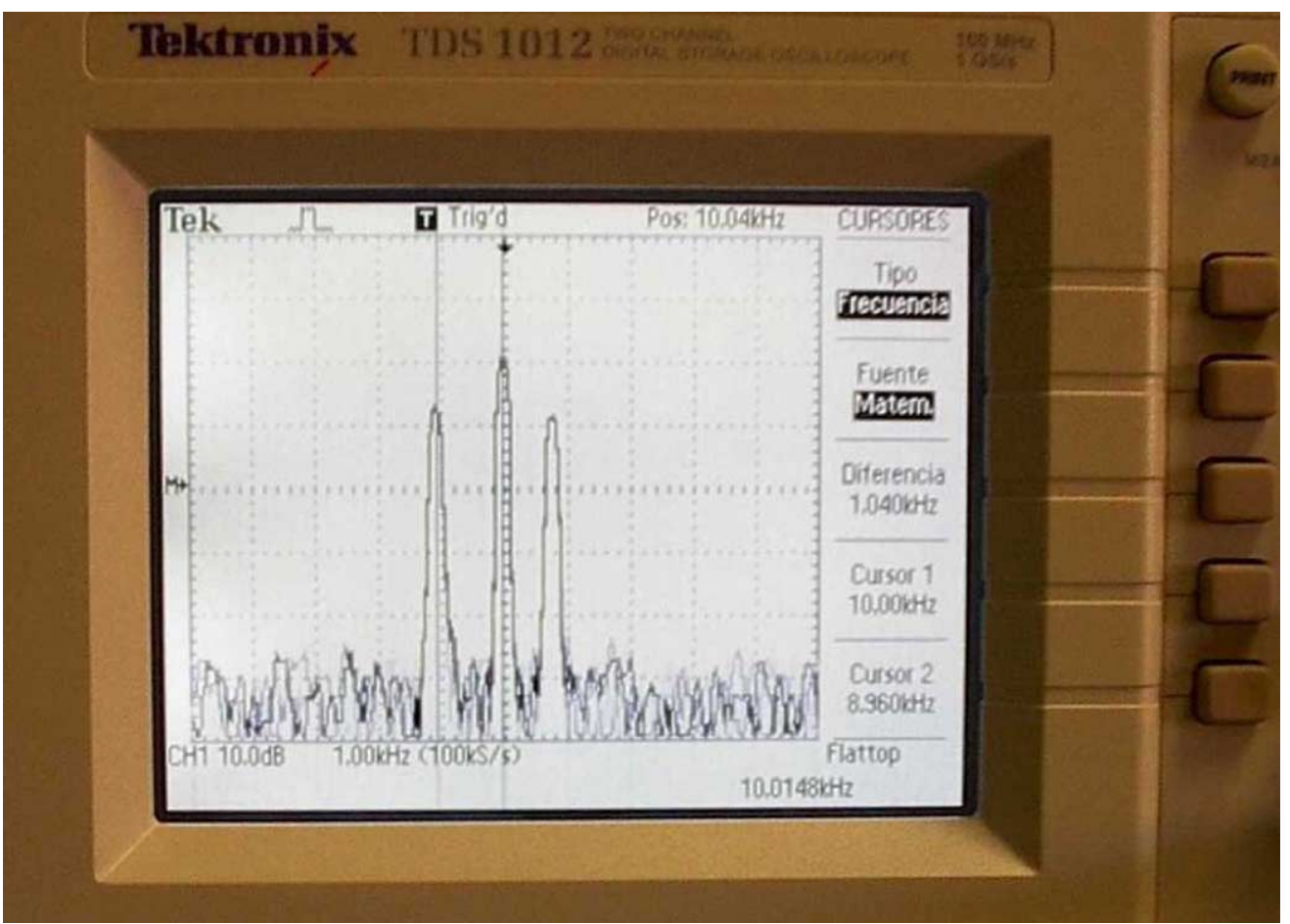

Fig. 10. Measurement of the spectrum of the output signal when three sensor heads are multiplexed. 
measured curve mean value, being this percentage of $7 \%$ in the second case. These quantities give us an idea of the good fit quality. The beam divergence is a low value in both sensors $<0.02 \mathrm{rad}$ (according to reality). And the constant, $k$, varies slightly for one sensor to another, although the same liquid, water, is used in both calibrations; so the differences are related to the POF attenuation, coupling coefficien in the different output of the POF coupler, ST-connectors and lens coatings. Relative measurements can be taken to avoid the $k$ influenc in the fina data. The experimental data used in the fittin process are taken for distances, from the lens to the liquid surface, which are greater than $20 \mathrm{~cm}$ and go up to $2 \mathrm{~m}$, this last value because of set-up limitations.

The theoretical study of this model provides the guide lines to improve the sensor head design. The main parameters, which influenc in the sensor response, are $-s$ the distance between the fibe centers; $a_{1}$ the lens radius; $f$ the focal length; $\alpha$ the beam divergence.

The constant $k$ will be considered as a multiplicative factor. Beginning with the parameter values used in the prototype (for $a_{1}, f$ and $s$ ) and the values coming from the curve fi $(\alpha)$, it has been studied the effects over the sensor response caused by the modificatio of their values. In each simulation, only one parameter has been modified keeping the others constant and equal to their original value. It has been tested that the new parameter values are inside the validity range of the model.

If the distance between the center of the fiber is increased, then the sensor response decreases. However, for $s<3 \mathrm{~mm}$ for the big sensor head, and $s<2 \mathrm{~mm}$ for the small sensor head, the sensor response does not depend on $s$. The reason for this result is that for these values of the parameter the behavior of the sensor is described for Eq. (5), where $s$ has no influence

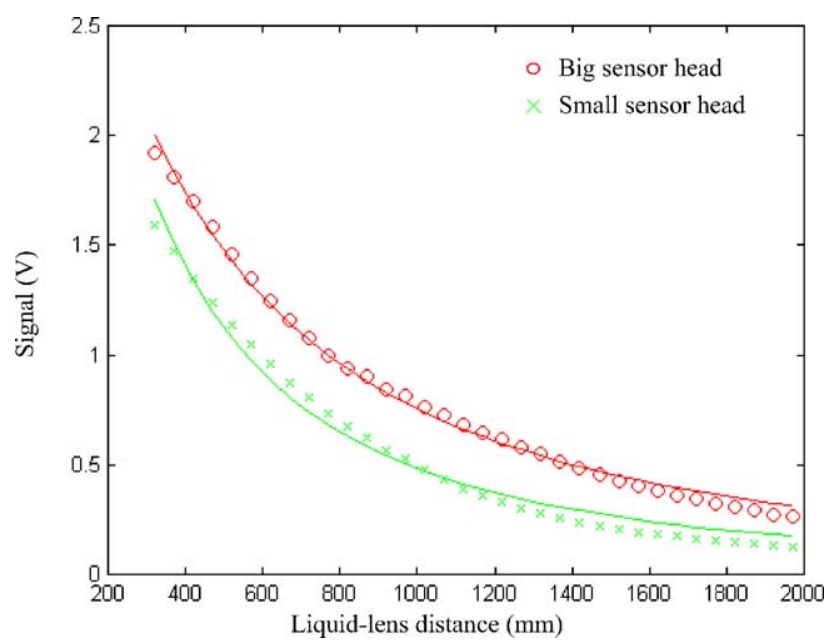

Fig. 11. Experimental data: $(\times)$ small sensor head; $(\bigcirc)$ big sensor head. (一) Curve fitte with Monte-Carlo method with: big sensor $2 a_{1}=60 \mathrm{~mm}$, $d_{\mathrm{m}}=0.031, \alpha=0.020 \mathrm{rad}, k=4.05$; small sensor $2 a_{1}=25.4 \mathrm{~mm}$, $d_{\mathrm{m}}=0.052, \alpha=0.014 \mathrm{rad}, k=5.01$.

As it has been experimentally observed (see Fig. 11), the bigger the lens radius is, the bigger the response of the sensor is. This fact is also reproduced by the proposed model.

For the considered parameters the focal lens does not influenc on the sensor response. Again, for these parameters, the Eq. (5), where the lens focal has no roll, controls the sensor behavior. However, the use of lenses of longer focal will allow to use lenses of bigger radius, increasing then the sensor response.

The last parameter considered is the beam divergence. $\alpha$ has been varied from 0 rad (perfectly collimated beam) to $0.1 \mathrm{rad}$ (beam with fi e times more divergence than the value obtained from the fit) It can be seen from Fig. 12 that if the

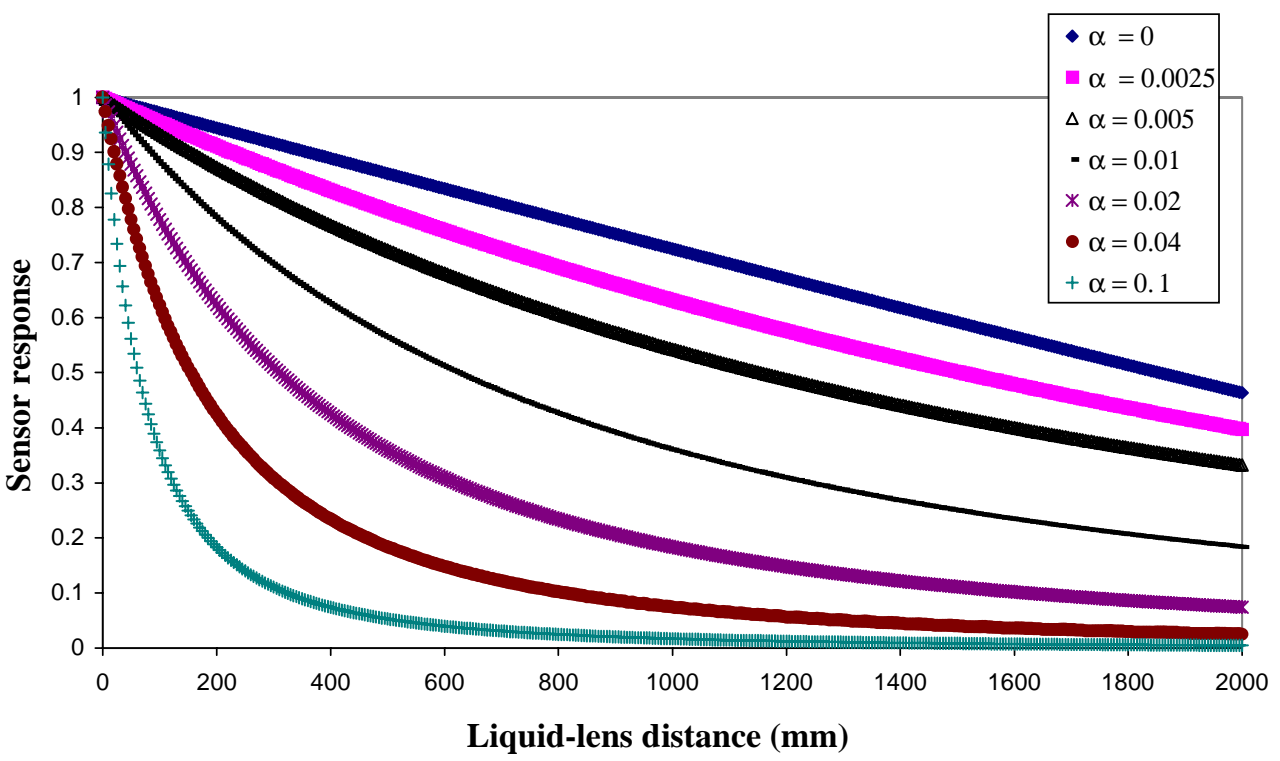

Fig. 12. Sensor response dependence on $\alpha . f=75 \mathrm{~mm}, s=0.98 \mathrm{~mm}, a_{1}=30 \mathrm{~mm}$. 


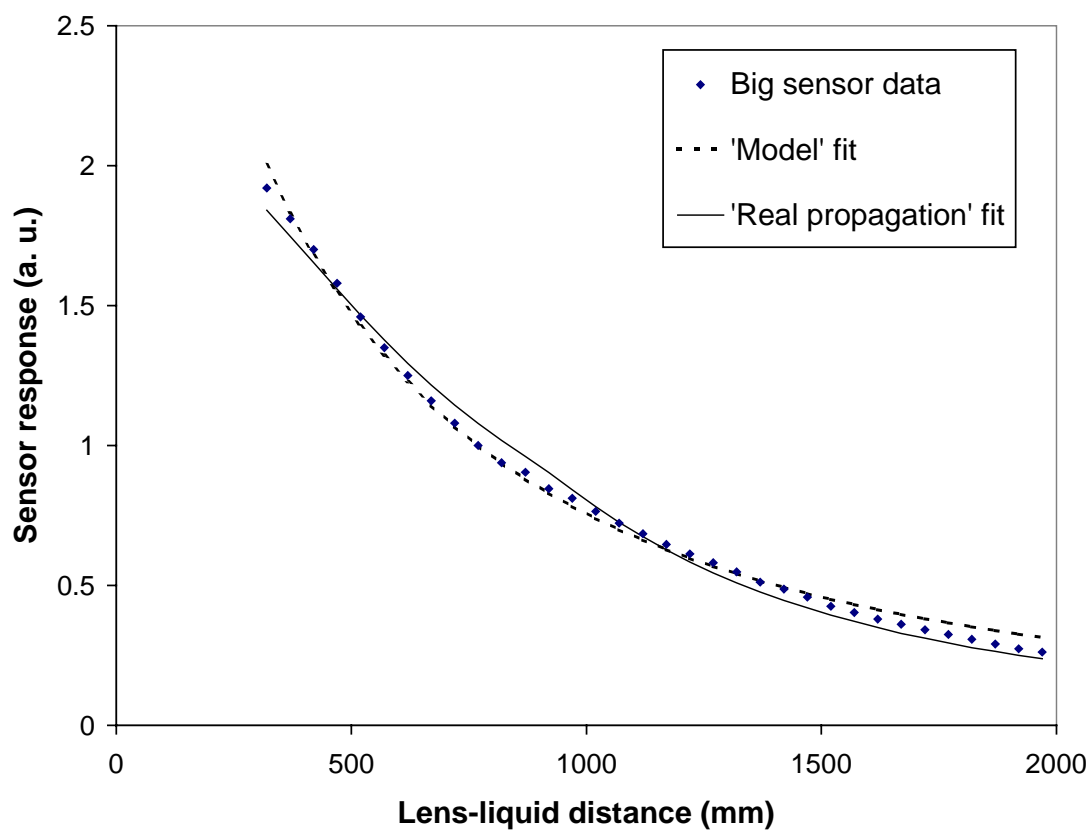

Fig. 13. Comparison between the experimental data (dots), the theoretical data without aberrations (---), and the theoretical data with aberrations (-).

divergence increases, the sensor response decreases and the curve is less similar to a line.

In principle, a sensor configuratio in which the beam was perfectly collimated will produce a bigger and more linear sensor response. However, in this case, the sensor is more sensitive to the modificatio of his parameters. For instance, his response will depend on the focal length and on all the values of $s$, that is, the tolerance range for these two parameters will disappear.

\subsection{Lens aberrations}

By means of the real propagation beam simulation, as it is described in Section 2.2, a curve for the sensor response was obtained, calculating the ratio $R_{2} / R_{1}$ for each lens-liquid distance, and multiplying the resulting distribution by a constant, which gives the minimum mean distance between the experimental and calculated data. The comparison of this curve not only with the experimental data, but also with the distribution obtained with the simple model, would give an estimation of the influenc of aberration in the sensor behavior. In Fig. 13 it is shown all the three mentioned curves. The two fit are similarly good. The mean distance between the experimental data and the data calculated with the simple model is 0.031 , as it can be seen in Table 1 . The mean distance between the experimental data and the data calculated taking into account the aberrations is 0.042 , that is, a value slightly higher. Hence, the inclusion of aberrations in the model of the sensor does not improve significantl the fit

\section{Discussion}

The proposed fiber-opti liquid level sensor is attractive because of its intrinsically safe and non-invasive nature. On the other hand, it has the problem that tilts (as mathematically described in Section 2.3) can influenc the sensor response. It is important to point out that the developed sensor can be used for sensing liquids with slow level variations (big tanks or pools) or after leaving the liquid surface to be stabilized. Having in mind that liquid surface tilts less than the half value of the beam divergence, $\alpha$, do not influenc the sensor response and that the sensor head with the big lens is less sensitive to these effects. Anyway, in mobile tanks with great tilts and ripples it is mandatory to use other type of liquid level sensors as those reported in [3,8,9], although they must be placed inside the liquid so wetting of the sensor can alter its performance and their range is limited to the sensor dimensions.

Reflectanc at the liquid surface can change and this will modify the constant $k$ and the sensor response. Periodic calibrations at a certain level can overcome this problem. Intensity fluctuation of the power source are overcame in the present prototype using an stabilized source, another alternative is using a POF splitter in the input fibe as a self-reference technique. For security purposes and to avoid the influenc of partial mobile spots in the liquid surface, more than one sensor head can be placed in a tank and comparison between their measurements can be performed.

The observed beam shape is very close to a circle so the assumption that the beam has an intensity circular pattern is quite good. 
In terms of multiplexing, different techniques can be used [18]. The FDM technique developed in this work along with the use of passive components to combine all the receiving fibers allows to simplify the electronics at the reception. In the level sensor reported in [9] a wavelength multiplexing technique (WDM) is proposed. In doing so, optical filter are necessary to address each sensor with a specifi wavelength. The expense and complexity of the WDM system gets worse with the number of channels and the consequent reduction in the wavelength separation, although it can be useful in fibe grating level sensors. Other approach is developing time division multiplexing using optical switches [19], in that case two sensor heads can be driven with a single laser source.

Finally we think that the model could be improved taken into account other effects, listed according to their importance as follows:

- the non-homogeneous spatial distribution of the laser power;

- the partially diffuse character of the liquid surface;

- the numerical aperture of the receiving fiber

- the air absorption of the radiation, etc.

\section{Conclusions}

In summary, we have developed novel fiber-opti liquid level sensor heads for a range greater than $2 \mathrm{~m}$, along with a theoretical model describing their behavior. Monte-Carlo method is used to fi the model parameters for two sensor heads with a good accuracy between the measurements and the fitte curve. The simple model has helped to optimize the design.

The non-contact sensor used a single lens for the emitting and receiving fibe and it is based on amplitude variations as a function of the liquid distance and not in time of fligh or phase detection. Being the firs fiber-opti liquid level sensor with those characteristics for long distances. These sensor heads are made of inexpensive, easy to handle POF and are used to develop a scalable system, intrinsically safe, for measuring the level in different tanks in flammabl atmospheres. The output signals are optically multiplexed and transmitted to a PC located in the central office An user-friendly interface is developed to show in real time the level in the different tanks and alarms activation in case of surpassing some predetermined levels. The multi-sensor system is developed for multiplexing eight sensors and it has been tested using three different sensor heads, with a $3 \times 1$ POF coupler and ST-connectors for optically multiplexing the output signals. These sensors exhibit a good linearity better than $1.5 \% \mathrm{FS}$ in a $2 \mathrm{~m}$ range. This range is limited by the present test-bench. Resolution better than $0.5 \%$ FS is obtained with an 8 bit $\mathrm{AD}$ converter and can be improved employing a better conditioning. A complex model including the aberrations is developed and it is shown that the fi is not significantl improved. Tilts of the liquid surface are also modeled and it is shown that if they are less than the half value of the beam divergence, there is no influenc on the sensor response. In applications demanding a good immunity to tilting and ripple effects, another type of sensors should be used.

\section{Acknowledgement}

This work has been partially supported by CICYT (TIC2003-03783) and CAM (07T/0011/2001) The authors gratefully acknowledge the contributions of J. Garcinuño and J.M.S. Pena in the prototypes.

\section{References}

[1] A.D. Kersey, A. Dandridge, Applications of fiber-opti sensors, IEEE Trans. Comp. Hybrids Manufact. Technol. 13 (1990) 137143.

[2] R. Clark, Laser liquid level gauge with diffuser, US Patent 5648844 (15 July 1997).

[3] S.W. James, S. Khaliq, R.P. Tatam, A long period grating liquid level sensor, in: Proceedings of the Optical Fiber Sensors Conference Technical Digest (OFS2002), vol. 1, 2002, pp. 127-130.

[4] K. Iwamoto, I. Kawata, Liquid-level sensor with optical fibe, Appl. Opt. 31 (1992) 51-54.

[5] L. Yuan, Automatic-compensated two-dimensional fiber-opti sensor, Opt. Fiber Technol. 4 (1998) 490.

[6] R. Felgenhauer, et al., Fiber optic displacement sensor with POF, in: Proceedings of the Plastic Optical Fiber Conference POF93, 1993, pp. 171-173.

[7] V.A. Svirid, V. León, S.N. Khotiaintsev, A prototype fiber-opti discrete level-sensor for liquid propane-butane, IEICE Trans. Electron. E83-C (2000) 303-308.

[8] G. Betta, A. Pietrosanto, A. Scaglione, A Gray-Code based fibe optic liquid level sensor, IEEE Trans. Instrum. Meas. 27 (1) (1998) 174-178.

[9] J.F.A. Morris, C.R. Pollock, A digital fiber-opti liquid level sensor, J. Lightwave Technol. LT-5 (7) (1987) 920-925.

[10] S.F. Knowles, B.E. Jones, S. Purdy, C.M. France, Multiple microbending optical-fibe sensors for measurement of fuel quantity in aircraft fuel tanks, Sens. Actuators A 68 (1998) 320323.

[11] A. Wang, M. Gunther, K. Murphy, R. Claus, Fiber-optic liquid level sensors, Sens. Actuators A 35 (1992) 161-164.

[12] P. Raatikainen, I. Kassamakov, R. Kakanakov, M. Luukkala, Fiber-optic liquid-level sensor, Sens. Actuators A 58 (1997) 9397.

[13] C. Yang, S. Chen, G. Yang, Fiber optic liquid level sensor under cryogenic environment, Sens. Actuators A 94 (2001) 69-75.

[14] B. Clifford, Remote sensor head for laser measurements devices, Patent WO99/56093 (29 March 1998).

[15] J. Zubia, O. Aresti, J. Arrue, M. Lopez-Amo, Barrier sensor based on plastic optical fibe to determine the wind speed at a wind generator, IEEE J. Select. Top. Quant. Electron. 6 (2000) 773779 .

[16] C. Vázquez, J. Garcinuño, J.M.S. Pena, A.B. Gonzalo, Multi-sensor system for level measurements with optical fibers in: Proceedings of the 28th Annual Conference of the IEEE Industrial Electronics Society, Sevilla, Spain, November 5-8, 2002. 
[17] C. Vázquez, Sistema sensor óptico para medida de nivel de líquidos en entornos críticos, Spanish Patent P200002032 (9 August 2000).

[18] E. Udd, Fiber Optic Smart Structures, Wiley, 1995.

[19] C. Vázquez, J.M.S. Pena, A.L. Aranda, Broadband $1 \times 2$ polymer optical fibe switches using nematic liquid crystals, Opt. Commun. 224 (2003) 57-62.

\section{Biographies}

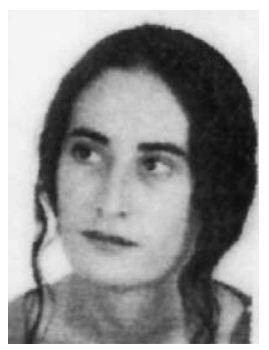

C. Vázquez (M'99) was born in Madrid, Spain, in May 1968. She received her MS degree in Physics (Electronics) in 1991 from Complutense University of Madrid, and her $\mathrm{PhD}$ degree at Telecommunications Engineering School in 1995 from Polytechnic University of Madrid (UPM). In 1991, she enjoyed fellowship at TELECOM (Denmark), working in erbium doped fibe amplifiers From 1992 to October 1995 she worked at Optoelectronics Division of "Telefónica Investigación y Desarrollo" in Madrid. She was involved in III/V integrated optics devices characterization, design and fabrication. In October 1995, she joined Carlos III University where she is currently working as an associate professor in the Electronics Technology Department.

Her current work includes design of fibe optics, LC and integrated optic devices and their application to optical communications, instrumentation and sensor networks. She is a member of the IEEE, SPIE, OSA, SEDO.

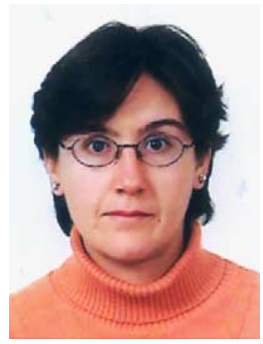

A.B. Gonzalo was born in Valladolid, Spain, in August 1974. She received her MS degree in Physics (General) in 1997 from the Universidad de Valladolid, and her $\mathrm{PhD}$ degree in Physics (Optics) in 2001 from the Universidad de Valladolid. She received a grant from MEC of Spain (AP97-09343565) to do this work. Since 2001 she has been an assistant professor in Electronics from the Universidad Carlos III de Madrid. She has worked in several projects in the area of Plasma Physics and in Vision Optics. At present, her research activity is centred on electro-optics, in special, in the application of optical fibe to the development of level sensors.

S. Vargas was born in Panamá, in 1973. He received his MS degree in Electrical \& Electronic Engineering in 1996 from Technological University of Panamá. He is an assistant professor at Electronics Technology Department of Carlos III University of Madrid. Currently, he is working towards a doctoral degree on designing of fibe optics and LC devices for their application to optical communications, mainly to WDM filters

J. Montalvo was born in Madrid, Spain, in May 1979. He received his MS degree in Telecommunication Engineering in January 2003 from Polytechnic University of Madrid (UPM). In March 2003, he joined Electronics Engineering Department at Carlos III University of Madrid (UC3M). As a member of the Displays and Photonic Applications Group, his research work is involved in fibe optic filter and devices characterization and design for Coarse Wavelength Division Multiplexing (CWDM) metro networks. 